\title{
Local reconstruction algorithms in the cathode strip cham- bers of CMS
}

\author{
Mirena Paneva ${ }^{1, *}$ on behalf of the CMS Collaboration \\ ${ }^{1}$ University of California, Riverside (USA)
}

\begin{abstract}
The design of the CMS detector is optimized for muon measurements. The muon system consists of gas ionization detector technologies. Cathode Strip Chambers (CSC) with both tracking and triggering capabilities are installed in the forward region. The first stage of muon reconstruction uses information from individual muon chambers and is thus called local reconstruction, in contrast to a subsequent global reconstruction where the information from all detectors is combined. First, 2-dimensional spatial points (rechits) describing where a muon crosses the CSC layers are built from the electrical signals induced by the charged particle traversing the chamber. Next, from the reconstructed hits, straight-line track segments are built within each chamber. Local reconstruction becomes particularly challenging at high instantaneous luminosities, which are expected at the HL-LHC. The high rate of particles traversing the detectors leads to increased rate of spurious rechits and segments thus increasing the combinatorial backgrounds. In this respect, work on improving the current and developing new algorithms is essential and is in progress. This document presents the existing local reconstruction algorithms used in the CMS cathode strip chambers. Their performance as well as ongoing efforts towards HL-LHC improvements are discussed.
\end{abstract}

\section{Introduction}

The Cathode Strip Chambers (CSC) provide precise tracking and triggering of muons in the forward region (endcap) of CMS and constitute an essential part of the CMS muon measurements [1]. CMS has 540 CSCs labelled ME for "Muon Endcap", with varying size depending on the location (Figure 1). An individual CSC consists of six layers of cathode strips and anode wires (Figure 2, left), each layer measuring the muon position in two coordinates, $r-\phi$. A charged particle passing through the chamber and ionizing the gas volume, induces charges on the strips and wires (Figure 2, right). The strips run radially outward and provide a precise measurement of the azimuthal coordinate. The wires are oriented perpendicular to the strips and provide a coarse measurement in the radial direction.

Raw data from the detector are unpacked into integer-based "digis" representing the strip and wire signals. The digis are used to produce a collection of objects called "rechits" representing the measurement of the intersection point between the traversing track and a CSC layer. The rechits reconstructed in each chamber form a segment, which is fitted to a straight line that provides a measure of the muon track trajectory in the chamber.

\footnotetext{
*e-mail: mirena.paneva@cern.ch
} 
Local reconstruction takes place at the level of individual chambers and denotes the sequence of steps leading from raw data to the first-level reconstructed objects (rechits and segments), which serve as input to the muon track reconstruction [2].

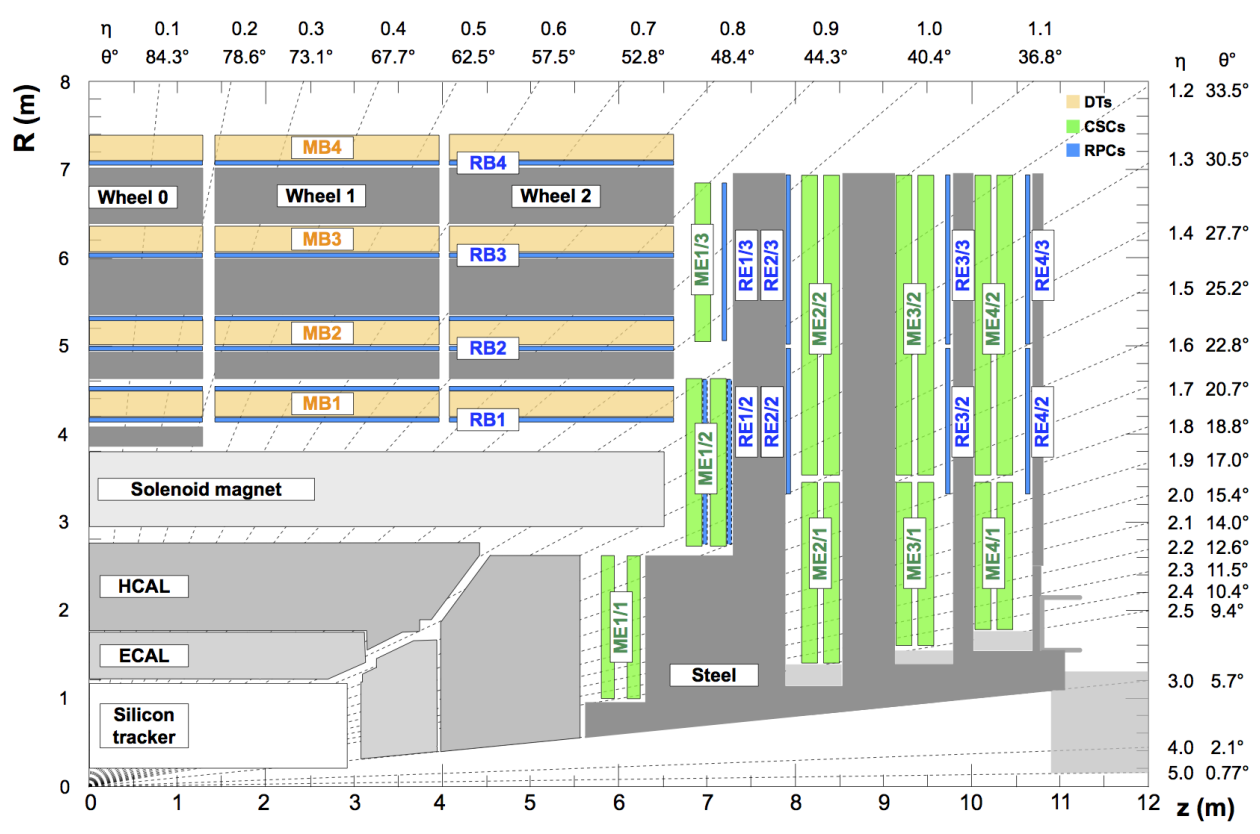

Figure 1. $R-z$ projection of one quadrant of CMS in the global coordinate system of the detector [1]. The horizontal axis is parallel to the beam and the interaction point is at the lower left corner.
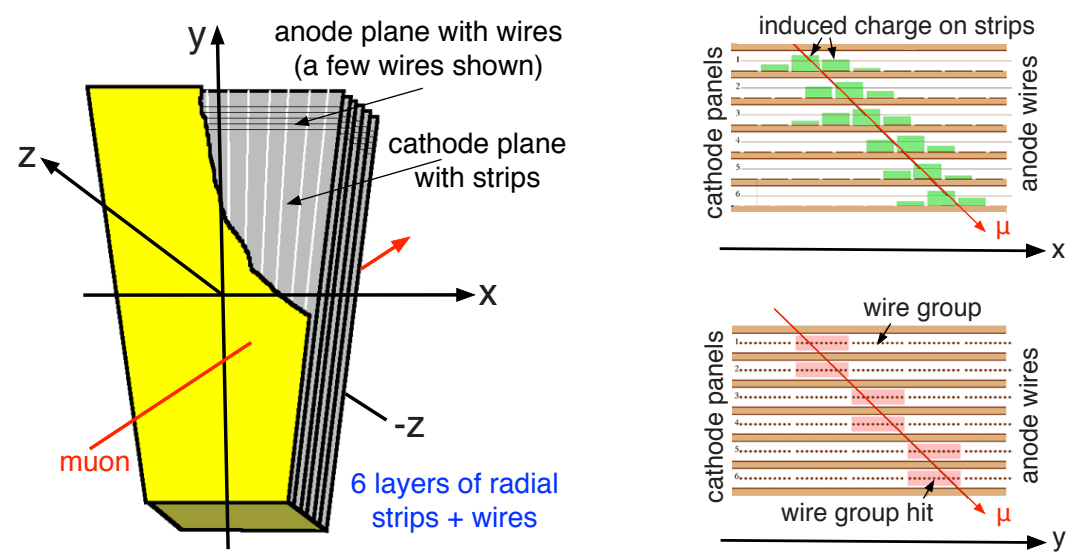

Figure 2. Left: Schematic view of a CSC. Right: Induced charges on strips and wire groups left by a muon. The coordinates $(x, y, z)$ are the local coordinates within an individual chamber with the origin of the coordinate system at the center of the chamber and the $z$-axis passing through the centre of each layer. 


\section{Reconstruction of hits}

A rechit describes the point $(x, y)$ where a muon traverses a layer of a CSC. The $y$ coordinate of the rechit is assigned according to the center-of-gravity of neighboring wire group hits. In principle, $x$ is obtained by the center of the charge distribution in a group of hit strips. In practice, the strip pulse-heights are sampled in $50 \mathrm{~ns}$ time bins, and the information from the peak of the pulse-height snapshots in time for each strip is used. The functional form describing the charge distribution in the cathode plane is known as a Gatti function [3], shown in Figure 3 , left. A Gatti function is fitted to the measured pulse-height peaks in the cluster of hit strips in the plane to provide the $x$-coordinate. In the CMS software, however, a parametrization of the induced charge distribution is used instead of a fit. This approach provides equally good results, but is faster and more robust [4]. For each strip in a cluster, three time samples are added together, as illustrated in Figure 3 (center). The measured charges $Q_{l}, Q_{c}, Q_{r}$ in left, central and right strips, respectively, are used to build a ratio $r$ defined as

$$
r=\frac{1}{2} \frac{Q_{r}-Q_{l}}{Q_{c}-\min \left(Q_{r}, Q_{l}\right)} .
$$

Figure 3 (right) shows the ratio $r$ as a function of the $x$-coordinate for different strip widths $w$. The $x$-coordinate of the cluster is then calculated via a function $x=f(r, w)$ that corrects for nonlinearity between $r$ and $x$. The parametrization function is derived in two steps. First, an empirical function is used to add a correction to $r$ in order to obtain the $x$-coordinate, where $x$ is based on the Gatti function as shown in Figure 3. A second empirical function is used to apply a correction due to the fact that the induced charge distribution does not quite follow the theoretical Gatti function. This second-order correction is obtained from cosmic rays data. The uncertainty in the position is also calculated from a parameterization, which is tuned for various kinematic and chamber geometries.
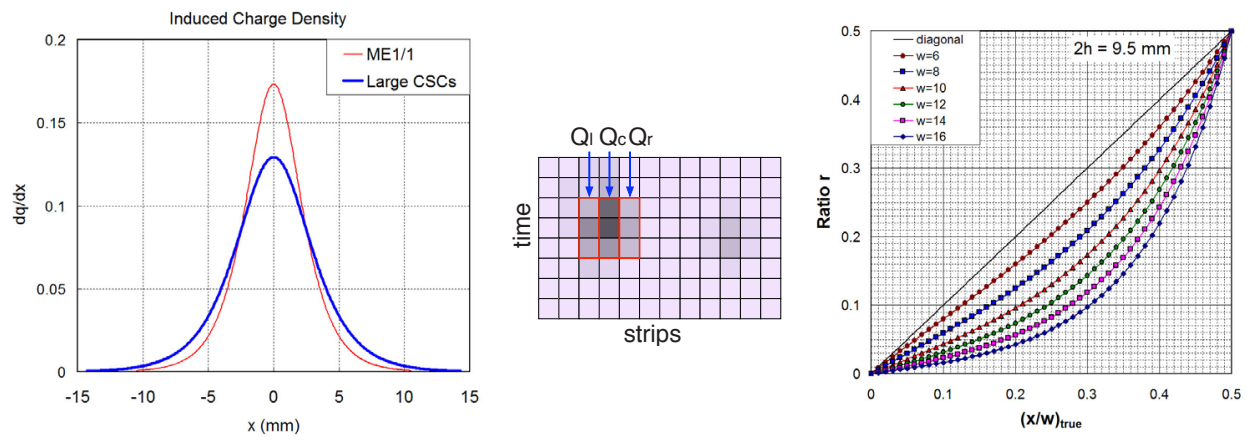

Figure 3. Left: Induced strip charge distribution according to a Gatti function. Center: Illustration of strip charge snapshots in time used to define the ration $r$ in Equation 1. Right: The ratio $r$ vs $x$ coordinate (in strip width units) for various strip widths $w$ (in millimeters) assuming a Gatti distribution [4].

\section{Reconstruction of segments}

For each CSC, the collection of reconstructed hits serves as input to a segment-building algorithm, which finds straight-line track stubs across the chamber using the available rechits from each of the 6 layers. A segment is effectively a set of 3-6 hits fitted to a straight line. Two such algorithms used in the CMS muon reconstruction software are discussed below. 


\subsection{Spanning Tree (ST) algorithm}

The Spanning Tree algorithm was used in the CMS software during the 7 and $8 \mathrm{TeV}$ running and part of the $13 \mathrm{TeV}$ running of LHC. It is based on pattern recognition using all hit combinations within a chamber. In the first step of the algorithm (pre-clustering), hits are grouped based on their $(x, y)$ coordinates via the "box" algorithm, as illustrated in Figure 4 (left), to form groups clearly separated from one another. In the segment finding step, each group of pre-clustered hits is fed to a "spanning tree" pattern recognition algorithm to check all combinations of hits within the group and find those that are most consistent with straight lines. The best segment candidates are chosen using variables sensitive to the angular offsets between the lines connecting hits from neighboring layers, as shown in Figure 4 (right), and minimizing the parameter $A$, which characterizes the spread of the hits, defined as (for an example case of 6 layers with hits):

$$
A=\left|\theta_{12}-\theta_{23}\right|+\left|\theta_{23}-\theta_{34}\right|+\left|\theta_{34}-\theta_{45}\right|+\left|\theta_{45}-\theta_{56}\right|
$$

The segment candidates chosen via the pattern recognition algorithm are fitted to a straight line using a least-squares method. The final step of the algorithm (hit pruning) aims to improve the segment quality by checking if the removal of any single hit significantly improves the fit.
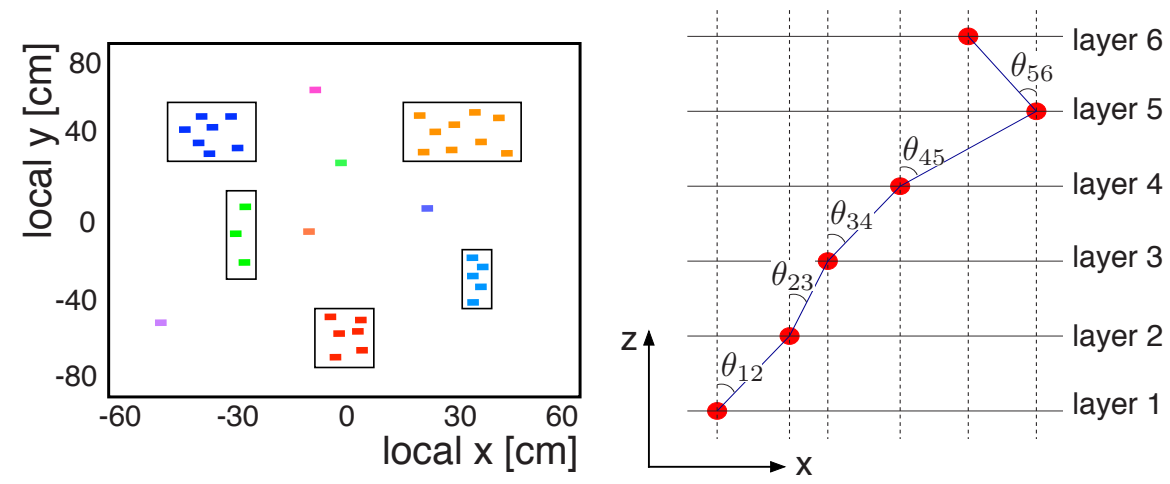

Figure 4. Illustration of the Spanning Tree algorithm. Left: Pre-clustering step. Right: Definition of the angles used for sorting criteria.

\subsection{Road Usage (RU) algorithm}

Beginning in 2017, a new segment reconstruction algorithm is used in the CMS software. The Road Usage algorithm [5] is faster and less complex than ST, and provides better performance at high luminosity where there is a large number $(\sim 35)$ of background interactions per beam crossing (pileup). The concept of the algorithm is schematically illustrated in Figure 5. First, the chamber layers with hits that are closest to and furthest from the CMS interaction point (shown in red) are taken as base layers. A pair of hits from the base layers is chosen such that the line connecting the two hits (shown in dashed magenta) points towards the interaction point. This requirement is an essential distinction with respect to the ST algorithm, and contributes to the reduction of fake segments with random orientation that are built out of background hits. Hits from the inner layers that are close to the "base road" (region shown in 
shaded blue) along the line connecting the base hits are added to the segment. If more than 1 hit in a layer satisfies the criteria, separate segments are built for each. After all possible segments consistent with the base layers are built, those that have common hits are grouped together. Each segment is fitted to a straight line. From each group only the segment with the lowest $\chi^{2}$ is chosen. If the segment does not pass a certain $\chi^{2}$ threshold, the hit furthest from the fit line is removed in order to minimize the sum of hit residuals, and the segment is refitted.

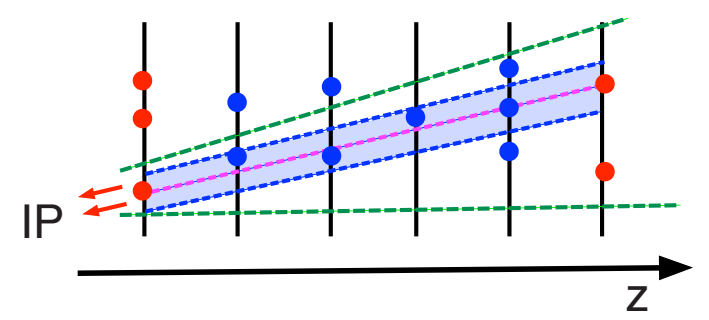

Figure 5. Schematic illustration of the Road Usage algorithm.

\section{Performance}

The performance of the local reconstruction algorithms is quantified in terms of efficiency and resolution, and has been studied with cosmic rays, collision data from LHC, and Monte Carlo simulated data $[1,6,7]$.

\subsection{Efficiency to find and associate a rechit to a segment}

The efficiency to find and associate a rechit to a segment is studied with simulated data. Rechits that are close to simulated hits in $(R-\phi)$ coordinates are selected. If more than one rechit is found in a layer, the closest one to the simulated hit is used. The efficiency is then defined as

$$
\text { efficiency }=\frac{\# \text { hits in segment }}{\text { \#hits in chamber }}
$$

As can be seen in Figure 6, the RU algorithm outperforms ST by a few percent for all chamber types, and the overall efficiency is close to $100 \%$.

\subsection{CSC spatial resolution}

For the measurement of the CSC spatial resolution, a data sample enriched in $Z \rightarrow \mu^{+} \mu^{-}$ events is used, and segments associated with reconstructed muons are selected. For each segment, one hit is dropped for a specific layer, the segment is refitted, and the residual for this hit is calculated. This is repeated for each layer, calculating a residual for each case. These residuals are combined to obtain the spatial resolution for each chamber. Resolution in the range of $40-150 \mu \mathrm{m}$ is measured, depending on the chamber type (Figure 6, right), which is well within the CSC design specifications. The difference in the resolution per chamber type is related to the strip sizes in the given CSC type. 

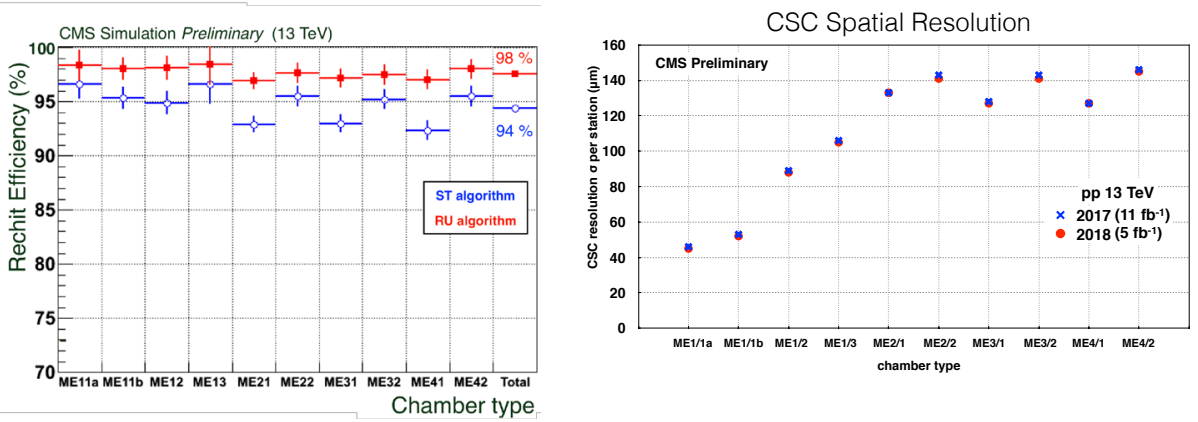

Figure 6. Left: Hit reconstruction and association efficiency. Right: Spatial resolution for the different CSC types.

\subsection{Segment reconstruction efficiency}

The efficiency to reconstruct a segment is measured using the Tag \& Probe technique in a data sample enriched in $Z \rightarrow \mu^{+} \mu^{-}$events. The tag is defined as a well-measured muon, the probe is a track reconstructed in the CMS tracking system and the invariant mass of the tag +probe is consistent with the $Z$ boson mass. The probe is projected into the CSC system and a matching segment is searched for in each CSC the track traverses. The measured efficiencies to reconstruct segments with the RU algorithm are shown in Figure 7 and are generally above 90-95\%. A few chambers out of 540 are known to be inefficient due to failed electronics boards.
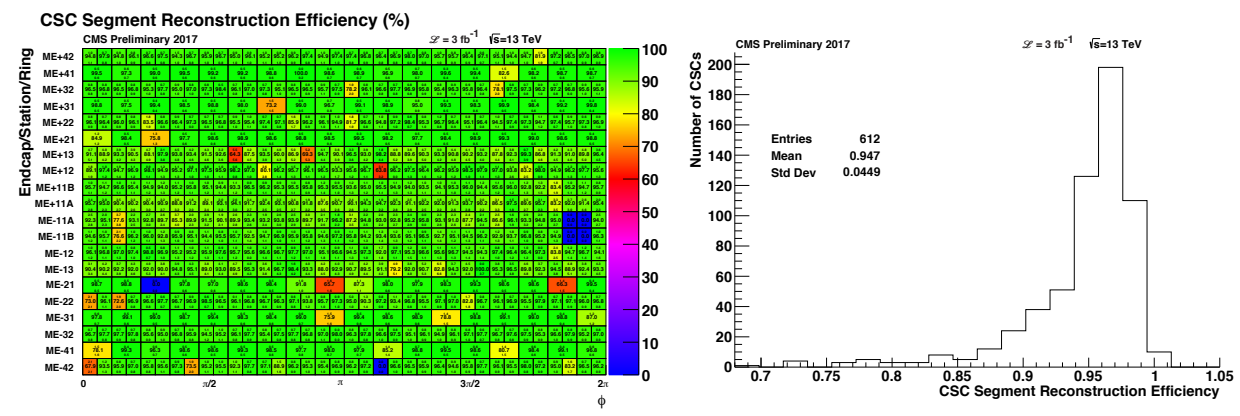

Figure 7. Segment reconstruction efficiency. Left: Detailed view with individual chambers. Right: Summary view.

\subsection{Segment properties and resolution}

Simulated data samples are used to study the properties and resolution of the segments reconstructed with the two algorithms. As demonstrated in Figure 8 (left), the RU algorithm tends to reconstruct fewer segments per chamber compared to ST, which contributes to a reduction of the combinatoric and spurious segments. The segments reconstructed with RU generally contain more hits (see Figure 8 (right)), which is beneficial for the segment quality. The resolution, estimated from the residuals between the reconstructed and the simulated segment 
position, is also improved with the RU algorithm. This effect is even more pronounced for highly energetic muons, as the comparison between muons with transverse momentum of $100 \mathrm{GeV}$ and $1000 \mathrm{GeV}$ demonstrates in Figure 9.
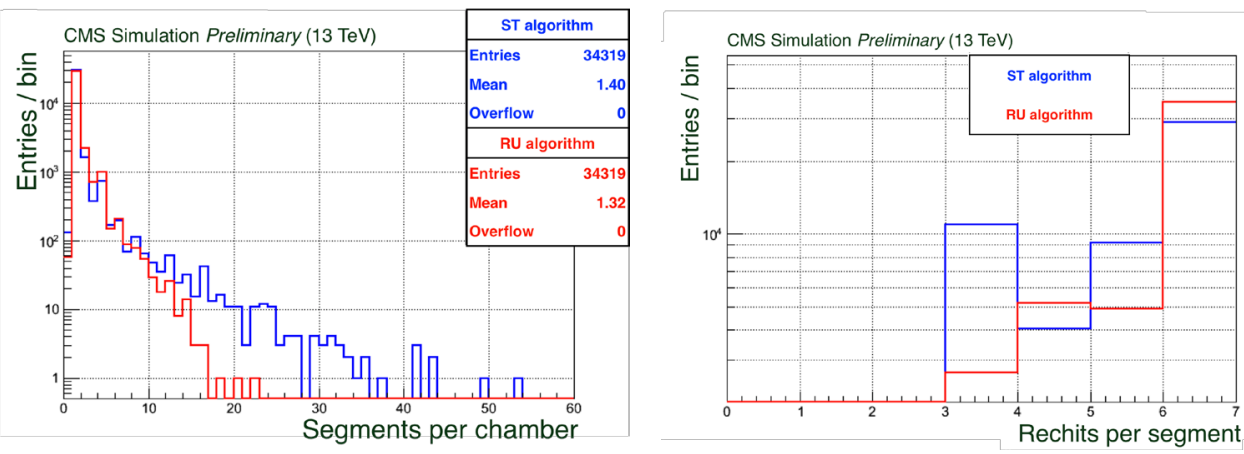

Figure 8. Performance comparison of the Road Usage and Spanning Tree algorithms for muons with a transverse momentum of $100 \mathrm{GeV}$. Left: Number of reconstructed segments per chamber. Right: Number of rechits associated to a segment.
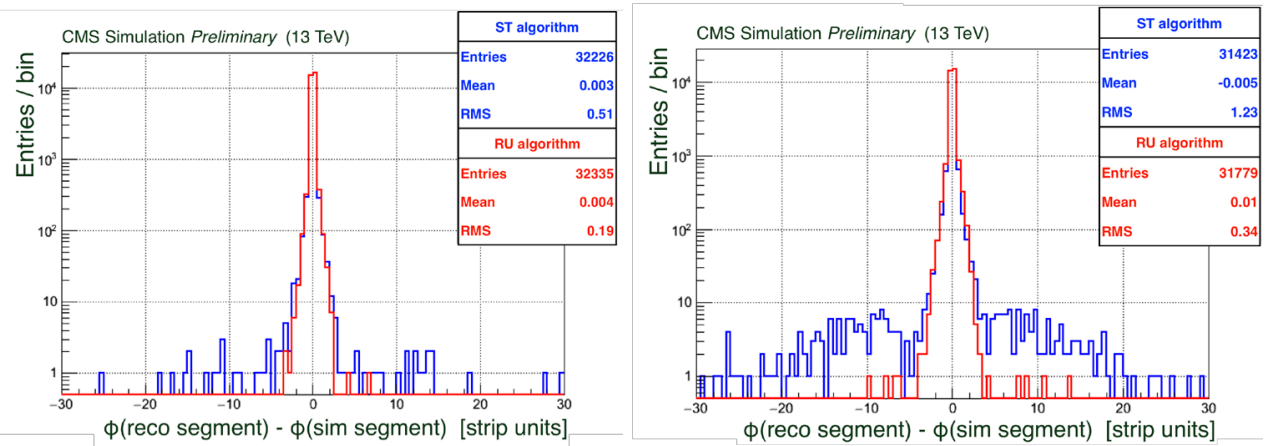

Figure 9. Segment resolution in $\phi$ estimated from the residuals between simulated and reconstructed segments for muons with a transverse momentum of $100 \mathrm{GeV}$ (left) and $1000 \mathrm{GeV}$ (right).

\section{Foreseen improvements}

The rate of CSC segments not originating from muons but from backgrounds, random hits and combinatorics, increases approximately linearly with the instantaneous luminosity of LHC. Because of the upgrade to HL-LHC, efforts are ongoing to improve the local reconstruction algorithms to meet the challenges of the new operation environment.

One possible improvement is the use of inverted logic for the segment reconstruction: instead of $1 \mathrm{D}$ wire/strip hits per plane $\rightarrow 2 \mathrm{D}$ rechits per plane $\rightarrow 2 \mathrm{D}$ segment(s) per chamber, construct 1D wire/strip hits per plane $\rightarrow 1 \mathrm{D}$ wire/strip segment(s) per chamber $\rightarrow 2 \mathrm{D}$ segment(s) per chamber. Potential benefits of this approach are pattern recognition that is less sensitive to random hits and additional handles to cope with combinatorial background. 
The latter include, among other studies, exploiting the strip cluster shape and correlations between wire and strip timing.

Another study aims at improving the hit reconstruction. Overlapping signals lead to a strip charge distribution that deviates from the Gatti functional form and produce a large uncertainty on the $\phi$-coordinate measurement ( $\gtrsim 30 \%$ of the strip width). Potential improvement of the hit resolution by utilizing Gaussian wavelets [8] is being studied.

Highly energetic muons with a momentum of $\geq 1 \mathrm{TeV}$ produce electromagnetic showers that increase the hit multiplicity in the CSCs. The number of hits per chamber can be more than 20, leading to a large number of possible segments. A potential solution for reconstructing such events is to use the center-of-gravity of the hits, and this is currently being investigated.

\section{References}

[1] CMS Collaboration, "Performance of the CMS muon detector and muon reconstruction with proton-proton collisions at $\sqrt{s}=13$ TeV”, JINST 13 (2018)P06015.

[2] G. Bruno et al., "Local Reconstruction in the Muon Detectors", CMS-NOTE-2002-043 (CERN, 2002).

[3] E. Gatti et al., "Optimum geometry for strip cathodes or grids in MWPC for avalanche localization along the anode wires", Nucl. Instr. and Meth. 163 (1979)83.

[4] V. Barashko et al., "Fast algorithm for track segment and hit reconstruction in the CMS Cathode Strip Chambers", CMS-NOTE-2007-023 (CERN, 2007).

[5] V. Palichik, N. Voytishin, "New CSC segment builder algorithm with Monte-Carlo TeV muons in CMS experiment", N. Phys. Part. Nuclei 48 (2017)786.

[6] CMS Collaboration, "Performance of the CMS Cathode Strip Chambers with Cosmic Rays", JINST 5 (2010)T03018.

[7] CMS Collaboration, "The performance of the CMS muon detector in proton-proton collisions at $\sqrt{s}=7 \mathrm{TeV}$ at the LHC", JINST 8 (2013)P11002.

[8] G. Ososkov, A. Shitov, "Gaussian wavelet features and their applications for analysis of discretized signals”, Comp. Phys. Comm. 126 (2000)149. 